\title{
A FULLY AUTOMATIC CALIBRATION PROCEDURE FOR FREEHAND 3D ULTRASOUND
}

\author{
François Rousseau, Pierre Hellier and Christian Barillot \\ IRISA, Université Rennes 1 - INRIA - CNRS \\ Campus de Beaulieu, \\ 35042 Rennes Cedex, France \\ Emails : $\{$ froussea, phellier, barillot $\} @$ irisa.fr
}

\begin{abstract}
This paper describes a novel method for calibration of freehand three-dimensional (3D) ultrasound. A position sensor is mounted on a conventional ultrasound probe, thus the set of B-scans can be localized in 3D, and can be compounded into a volume. The calibration process aims at determining the transformation (translations, rotations, scaling) between the coordinates system of images and the coordinate system of the localization system. In our study, the phantom used to calibrate the $3 \mathrm{D}$ ultrasound system is a plane. It provides in each image a strong, straight line. The calibration process is based on the set of lines in 2D images forming a plane in 3D. Points of interest are extracted from the ultrasound sequence. The eight parameters of the transformation are determined with an iterative algorithm which is based on the principle that correct registration between the plane and the points of interest provides correct calibration. Validation of this method has been performed on synthetic sequences. This calibration method is shown to be easy to perform, completely automatic and fast enough for clinical use.
\end{abstract}

\section{INTRODUCTION}

Nowadays, more than one out of every four medical imaging studies is performed with ultrasound. 2D ultrasound is popular because of its non-invasive nature, its real time capability and relatively cheap cost of acquisition. The purpose of three-dimensional ultrasound is to dismiss the main drawback of traditional two-dimensional ultrasound imaging which is its weak capability of issuing quantitative accurate morphometric information. Furthermore, 3D ultrasound facilitates extensive investigation and allows accurate measurements of organ volumes.

A 3D examination can be broken into three stages : acquisition of images, reconstruction and visualization. The acquisition stage is significant because it strongly determines the final results. Three solutions have been proposed to acquire images: freehand acquisition systems (manual sweeping), mechanical systems and three-dimensional probes. To acquire 3D images, it seems natural to modify classical probe to explore directly the patient in $3 \mathrm{D}$ with a $2 \mathrm{D}$ array. Altough this solution is a promising way for 3D ultrasound, 2D phased array of elements is still at an experimental stage. As a matter of fact, technical problems (interactions between the transducers, cumbersome interfacing, complex electronics, etc.) are currently a challenging subject of research. Finally, the last option is to automate the motion probe with a mechanical system. It produces sequences of regular echographic images of the patient. There are three types of mechanical movements : linear, rotational and fan. The three-dimensional reconstruction is then accurate, but these types of systems suffer from a lack of freedom of movement.

The technique used in this paper is a $3 \mathrm{D}$ freehand acquisition. The freehand solution consists in fixing a localization system on the probe, which continuously gives the position and orientation of the probe. The localization system can be magnetic, optic, acoustic or mechanical. We choose a magnetic system because this type of device provides a flexible and low-priced solution. Moreover, electromagnetic devices can be easily used for routine applications. This type of system have already been successfully widely used for 3D ultrasound (e. g. [1, 2, 3]).

The calibration consists in determining the relation between any point of the ultrasound image and its coordinates in the fixed reference mark related to the system of localization. It is an crucial step because it has a significant impact on the quality of reconstruction. However the calibration process is independent of the localization system.

When the calibration is performed, the $3 \mathrm{D}$ volume can be reconstructed. 3D surface models and voxel-based volume models are the most popular methods to reconstruct the $3 \mathrm{D}$ volume ([4]). Finally, the data may be visualized with many techniques : surface-based, volume-based, re-slicing, panoramic viewing, multi-planar.

This paper focuses on the calibration stage for freehand 3D ultrasound. We propose a new algorithm which is based on the hypothesis that correct registration between the geometry of the phantom and the points of interest provides 
correct calibration.

\section{CALibRATION ISSUES}

During the reconstruction, each pixel of each scan has to be localized in the reconstruction volume. It is thus necessary to determine the transformation between a pixel of the $2 \mathrm{D}$ image and a point in the reconstruction volume. In general, the calibration problem can be formulated in the following way ([3]):

$$
\underline{X}_{c}=T_{c} T_{t} T_{r} \underline{X}_{r}, \text { with } \underline{X}_{r}=\left(\begin{array}{c}
s_{x} u \\
s_{y} v \\
0 \\
1
\end{array}\right)
$$

where $T_{r}$ denotes the transformation from B scan to receiver coordinates, $T_{t}$ the transformation from receiver to transmitter coordinates, $T_{c}$ the transformation from transmitter to phantom coordinates, $u$ and $v$ column and row indices of the pixel in the image, $s_{x}$ and $s_{y}$ scaling factors $(\mathrm{mm} / \mathrm{pixel})$. Thus, the coordinates of each pixel are expressed first in the coordinate system of the receiver $R$, then to the transmitter $T$ and finally to the reconstruction volume $C$. The different coordinate systems are illustrated in figure 1.

In this formulation, we assume that the ultrasonic beam is a plane. This hypothesis can be done because beam thickness accounts for only some of the errors in real calibration. The RMS error is relatively insensitive to beam thickness ([3]).

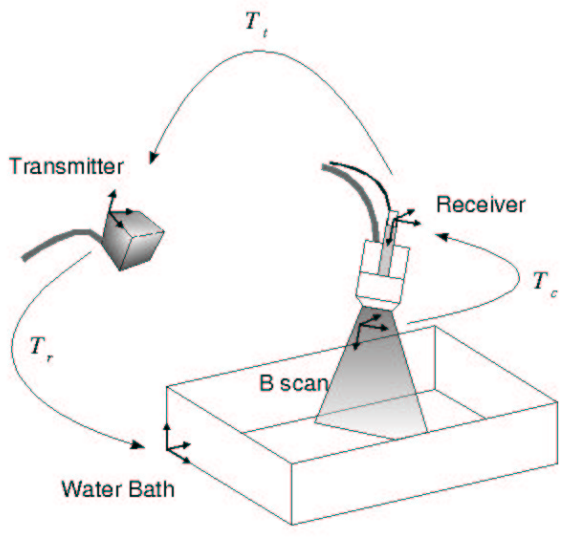

Fig. 1. Coordinate systems

The calibration amounts to estimating the matrix $T_{r}$ and the coefficients $s_{x}$ and $s_{y}$. We have to determine a rigidbody transformation with six parameters (three for translations and three for rotations) and two scaling factors. The calibration is generally carried out in the following way: after scanning a phantom whose 3D geometrical properties are known, the calibration is then based on this geometry being recovered in the sequence of ultrasound images.

Many phantoms like points $([1,2,5,6])$ or plane ([3]) exist and some studies ([3]) have been conducted to compare these phantoms. The cross-wire and the three-wire techniques suffer from relatively poor repeatability and extremely long calibration times. It has appeared ([3]) that using a flat plane produces lines which can be reliably and automatically detected.

Blackall et al. recently proposed [7] an image registration approach to calibrate freehand 3D ultrasound. The idea is that a consistent calibration gives an optimal similarity measure between the ultrasonic images of the phantom and a 3D model of this one, whose geometry is perfectly known. Registration is obtained by the maximization of normalized mutual information ([8]). The final results of precision and reproductibility are comparable with the method using point based phantom, but with a reduced time of image acquisition (2 minutes).

Finally, in [5] and [7], it is necessary to know the coordinates of the landmarks in the space of reconstruction. This can be done with a high degree of accuracy using an optical pen but this step is tedious and time-consuming.

No solution so far have been proposed to solve this problem in fully and fast automatic way. In order to meet the requirement of 3D ultrasound on routine applications, we aim at proposing a calibration technique which is easy to use, rapid, robust and completely automatic.

\section{METHOD}

With the aim of carrying out a simple, fast and completely automatic calibration, we have chosen to use a plane phantom. This type of phantom is very easy to use. Moreover, it provides in each image a strong, straight line which can be automatically detected with high accuracy.

Our algorithm is based on the principle that a correct calibration of the parameters gives an optimal registration of the ultrasonic images with the plane. In each image, we fist extract points belonging to the line produced by the water bath. To obtain these points, the gradient image of each Bscan is computed. The highest gradient points are retained. Points of interest have to be aligned with the plane. Let $R$ be the set of points of interest, given the equation of the plane, calibration parameters are estimated to minimize the euclidian distance between the plane and the points of R. Then, distance between the plane and each point of interest have to be computed and the function $f$ to minimize is :

$$
f\left(t_{x}, t_{y}, t_{z}, \alpha, \beta, \gamma, s_{x}, s_{y}, a, b, c, d\right)=\frac{1}{N} \sum_{M \in R} d(\pi, M)^{2}
$$


where $d(\pi, M)$ is the euclidian distance between the plane $\pi$ and the point $M$, and $R$ denotes the set of $N$ points used for the registration. $t_{x}, t_{y}, t_{z}$ are the translation parameters, $\alpha, \beta, \gamma$ are the rotation parameters, and $s_{x}, s_{y}$ are the two scaling factors. The euclidian distance between the plane $\pi$ and a point $M$ is given by :

$$
d(\pi, M)=a X_{i j}+b Y_{i j}+c Z_{i j}+d
$$

where $a, b, c$ and $d$ are the normalized planar coefficients. The coordinates of $M$ given by the vector $\left(X_{i j}, Y_{i j}, Z_{i j}\right)$ are expressed in the coordinate system of the transmitter.

Unlike [3], we do not use any fixed reconstruction volume (here, the water bath), then equation of the plane is given in the coordinate system of the transmitter. Therefore we have to estimate the coefficients $a, b, c$ and $d$. The plane $\pi$ has no particular equation in the coordinate system of the transmitter. So, from the equation (1), we can eliminate the matrix $T_{c}$.

Using this formulation, twelve parameters have to be estimated. Given an initial estimate of each parameter, the best parameters may be determined using a classical optimization algorithm. Since gradient of $f$ can be easily computed, the Levenberg-Marquardt algorithm is used to estimate the six parameters in the 3D transformation matrix, the two pixel scaling factors and the four plane coefficients.

\section{NUMERICAL ISSUES}

Our algorithm has been tested with synthetic sequences. A recurrent problem in medical imaging is that ground truth is never exactly known. Therefore it is interesting to evaluate a method with synthetic sequences where optimal parameters are exactly known. Thus, we can evaluate the precision and the repeatability of the calibration algorithm. Moreover, it is possible to test the intrinsic precision of our method, because with synthetic sequences, many types of errors are eliminated (localization errors of each B scan, errors due to beam thickness...).

The initialization stage of the algorithm is also an important part of the method that we have to evaluate. To test the influence of the initialization step, we initialize the algorithm with random values chosen with uniform law. For each parameter, a initialization domain has been defined with respect to known optimal parameters. For each test, the algorithm was tested with 40 starting estimates, with combinations of $\pm 20 \mathrm{~mm}$ and $\pm 20^{\circ}$ for the rigid transformation parameters, and $\pm 30 \%$ for the scaling factors.

To shorten the computation time, we propose an hierarchical algorithm. The complexity of the algorithm only depends on the number of points of interest used to compute the minimisation of $f$. Classically, to shorten computation time, a multiresolution algorithm is used. With the same idea, we can divide the optimization part in three different steps. With a fixed number $N$ of points of interest, the first optimization part is done with a third of $N$ points, then the second part with two thirds of $N$ points, and finally, with all the extracted points.

\section{RESULTS}

The method was tested with three synthetic sequences of 61 images. In each case, the size of the image is 256 by 256 (pixels). Ultrasound images are classically corrupted by the presence of speckle noise. To test the algorithm with realistic sequences, we add speckle noise to images. Speckle can be modeled with the Rayleigh law. The Rayleigh probability density function is defined by the following equation :

$$
f(x)=\frac{x}{\sigma^{2}} e^{-\frac{x^{2}}{2 \sigma^{2}}}, x \geq 0
$$

Speckle is generally modeled as a multiplicative noise : $I_{2}=n I_{1}$, where $I_{1}$ is the original image, $I_{2}$ is the corrupted image and $n$ is the speckle pattern. As speckle noise is a multiplicative noise, we add noise to a gray background of the image where $\sigma$ is equal to 1 . Around the plane, we accentuate noise and $\sigma$ is equal to 10 .

Figure 2 illustrates speckle effect on the original sequence.
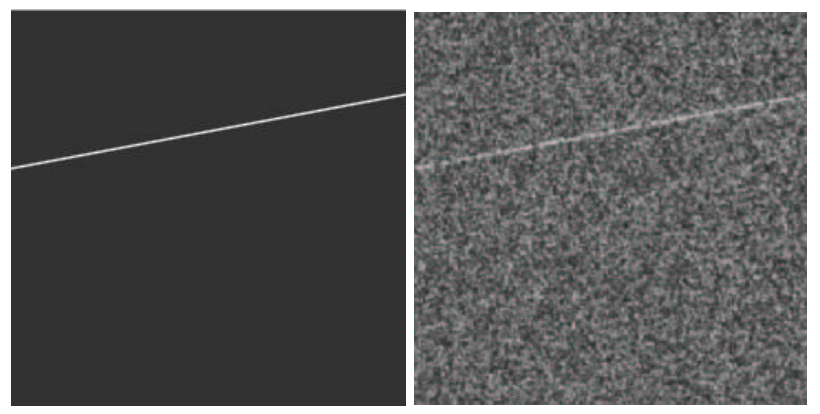

Fig. 2. Original and added speckle noise sequences.

\subsection{Complexity and computation time}

As mentioned above, the complexity of the algorithm depends on the number of points of interest used to compute the minimisation of $f$. The synthetic sequences are composed by 61 images. According to our experiments, it seems reasonable to take about five hundred points of interest for the whole sequence, which means ten points per image.

The computation time depends on the number of extrated points, the initialization domain and the desired precision in the optimization step. The computation time for the three synthetic sequences is reported in table 1 , and is 


\begin{tabular}{|c|ccc|ccc|c|}
\hline & \multicolumn{3}{|c|}{ Mean Error } & \multicolumn{3}{c|}{ Standard Deviation } & Mean Time \\
\hline Sequence 1 & 0.6546 & 0.1849 & 0.0040 & 3.2140 & 1.9963 & 0.0044 & 22.76 \\
\hline Sequence 2 & 0.5146 & 0.1473 & 0.0010 & 2.7600 & 1.6552 & 0.0109 & 31.26 \\
\hline Sequence 3 & 0.6143 & 0.1472 & 0.0020 & 3.0164 & 1.9749 & 0.0121 & 29.52 \\
\hline
\end{tabular}

Table 1. Calibration results : Mean error and standard deviation are given for translations, rotations and scaling factors. The results for translations are in $\mathrm{mm}$, for rotations in degrees, for scaling factors in $\mathrm{mm} / \mathrm{pixel}$ and for mean time in minute.

equal to approximatively 25 minutes in each case. The hierarchical decomposition of the optimisation step has divided the computation time by a factor of 2 . However, the algorithm has been tested with a quite large initialization domain to evaluate its robustness and the computation time is very dependent to the initialization domain. We have tested our algorithm with the same initialization domain as [7] and the computation time decreases to ten minutes.

\subsection{Precision and reliability of the algorithm}

To evaluate the quality of the results, the mean of the difference between the estimated parameters and the known optimal parameters, and the standard deviation are calculated. Mean error indicates the convergence of the algorithm to the solution and standard deviation indicates the reliability of the results. Calibration results are summarised in table 1 . These results indicate that even with noisy sequences and a large initialization domain, the calibration appears to be quite accurate. With the same initialization domain as [7], standard deviations for translations and for rotations are divided in half. These results show that our calibration method is accurate enough to be used in clinical applications.

\subsection{Comparison with Stradx results}

\begin{tabular}{|l|lll|}
\hline & \multicolumn{3}{|c|}{ Mean Error } \\
\hline Sequence 1 & 1.3833 & 0.9011 & 0.0095 \\
\hline Sequence 2 & 1.5979 & 0.5932 & 0.0056 \\
\hline Sequence 3 & 0.8672 & 0.1522 & 0.0036 \\
\hline
\end{tabular}

Table 2. Stradx calibration results : Mean error is given for translation $(\mathrm{mm})$, rotations $(\mathrm{deg})$ and scaling factors ( $\mathrm{mm} /$ pixel).

Finally, we have tested the three synthetic noisy sequences with the Stradx software, developped by [3] at Cambridge University. The results are summarised in table 2 . With the presence of fully-developped speckle in each sequence, line detection parameters in Stradx have to be tuned, otherwise Stradx algorithm could fail to converge. It would be unfair to compare directly our results with the results obtained with Stradx, since the latest strongly depend on the tuning of the line extraction parameters.

\section{CONCLUSION}

We present in this paper a novel fully automatic method for performing a calibration of a 3D freehand ultrasound system. The calibration parameters are determined using an iterative registration algorithm between a plane phantom and points of interest extrated in $2 \mathrm{D}$ images. The method has been shown to be robust, accurate and fast enough for clinical use.

\section{REFERENCES}

[1] P. R. Detmer, G. Bashein, T. Hodges, K. W. Beach, E. P. Filer, D. H. Burns, and D. E. Strandness Jr, "3d ultrasonic image feature localization based on magnetic scanhead tracking : in vitro calibration and validation," Ultrasound in Medicine and Biology, vol. 20, no. 9, pp. 923-936, 1994.

[2] J. Carr, Surface reconstruction in 3D medical imaging, Ph.D. thesis, University of Canterbury, Christchurch, New Zealand, 1996.

[3] R. W. Prager, Rohling R. N., Gee A. H., and Berman L., "Rapid calibration for 3-d freehand ultrasound," Ultrasound in Medicine and Biology, vol. 24, no. 6, pp. 855-869, 1998.

[4] A. Fenster, D. B. Downey, and H. N. Cardinal, "Threedimensional ultrasound imaging," Physics in medicine and biology, vol. 46, pp. 67-99, 2001.

[5] D. Henry, Outils pour la modélisation des structures et la simulation d'examens échographiques, Ph.D. thesis, Université Joseph Fourier - Grenoble 1, 1997.

[6] S. Meairs, J. Beyer, and M. Hennerici, "Reconstruction and visualization of irregularly sampled three- and four-dimensional ultrasound data for cerebrovascular applications," Ultrasound in Medicine and Biology, vol. 26, no. 2, pp. 263-272, 2000.

[7] J. M. Blackall, D. Rueckert, C. R. Maurer Jr, G. P. Penney, D. L. G. Hill, and D. J. Hawkes, "An image registration approach to automated calibration for freehand 3d ultrasound," in Proc. of Medical Image Computing and Computer-Assisted Intervention, 2000.

[8] C. Studholme, D.L.G. Hill, and D.J. Hawkes, "An overlap invariant entropy measure of $3 \mathrm{~d}$ medical image alignment," Pattern Recognition, vol. 32, no. 1, pp. 71-86, 1999. 